\title{
Using density functional calculations to elucidate atomic ordering of Pd-Rh nanoparticles at sizes relevant for catalytic applications
}

\author{
Lorena Vega a , Hristiyan A. Aleksandrov b,* , Konstantin M. Neyman a,c,\# \\ a Departament de Ciència dels Materials i Química Física \& Institut de Química Teòrica i Computacional, Universitat de Barcelona, 08028 Barcelona, Spain \\ b Faculty of Chemistry and Pharmacy, University of Sofia, 1126 Sofia, Bulgaria \\ c Institució Catalana de Recerca i Estudis Avançats (ICREA), 08010 Barcelona, Spain
}

\section{A R T I C L E I N F O}

\section{Article history:}

Received 2 March 2019

Accepted 6 April 2019

Published 5 November 2019

Keywords:

Density functional calculations

Atomic ordering

Pd-Rh nanoparticles

Size relevant

\begin{abstract}
A B S T R A C T
$\mathrm{Pd}-\mathrm{Rh}$ nanoparticles are known to easily undergo surface restructuring in reactive environment. This study quantifies, with the help of density functional (DFT) calculations and a novel topological approach, atomic ordering and surface segregation effects in Pd-Rh particles with compositions 1:3, 1:1 and 3:1 containing up to 201 atoms (ca. $1.7 \mathrm{~nm}$ ). The obtained data are used to reliably optimise energetically preferred atomic orderings in inaccessible by DFT Pd-Rh particles containing thousands of atoms and exhibiting sizes exceeding $5 \mathrm{~nm}$, which are typical for catalytic metal particles. It is outlined, how segregation effects on the surface arrangement of Pd-Rh nanoalloy catalysts induced by adsorbates can be evaluated in a simple way within the present modelling setup.
\end{abstract}

(C) 2019, Dalian Institute of Chemical Physics, Chinese Academy of Sciences. Published by Elsevier B.V. All rights reserved.

\section{Introduction}

A combination of $\mathrm{Pd}$ and $\mathrm{Rh}$ metals is present in the active component of modern automotive three-way catalysts [1,2]. Moreover, bimetallic Pd-Rh nanoparticles (NPs) are known to efficiently catalyse, beyond purification of car exhaust gases [3], reactions of $\mathrm{CO}_{2}$ methanation [4], methanol oxidation [5] and ethanol steam reforming [6], just to mention a few processes. Pd-Rh NPs reveal remarkably easy surface restructuring under reaction conditions due to interactions with oxidizing and reducing environments [7-9] as well as with oxide supports [10]. Notably, Pd and Rh immiscible at low temperatures in bulk [11-13] can mix in NPs [3-10,14].

Atomic-level understanding various peculiarities of catalytic $\mathrm{Pd}-\mathrm{Rh}$ NPs requires reliable information on their thermodynamically stable surface arrangements in the absence of per- turbations by reactants and/or supports. Obtaining such reference data experimentally is complicated by high sensitivity of the surface atomic ordering of Pd-Rh NPs to environment. This sensitivity resulted in a variety of surface arrangements [3-10] different from the ordering Pd-shell/Rh-core expected in vacuum for strongly surface segregated $\mathrm{Pd}$ on the basis of its lower surface energy compared to $\mathrm{Rh}$ [15] and in line with the surface segregation propensity of Pd impurity in Rh metal [16]. Hence, the knowledge about the detailed structure of such versatile entities as Pd-Rh NPs is crucial for tuning their properties in order to extend and make more efficient their catalytic applications.

Computational modelling of bimetallic nanoparticles using density functional theory (DFT) methods can provide valuable information on the structure-properties relations, complementary to experimental data. DFT calculations of bimetallic nano-

\footnotetext{
* Corresponding author. E-mail: haa@chem.uni-sofia.bg

* Corresponding author. E-mail: konstantin.neyman@icrea.cat

DOI: S1872-2067(19)63362-0 | http://www.sciencedirect.com/science/journal/18722067 | Chin. J. Catal., Vol. 40, No. 11, November 2019
} 
particles containing over hundred atoms $(\sim 1.5 \mathrm{~nm}$ large $)$ became feasible more than a decade ago [17]. Yet, the dramatically increased complexity of alloy NPs due to the presence of two types of metal atoms hinders their extensive computational studies. Two more degrees of freedom characterise a bimetallic particle $\mathrm{A}_{m} \mathrm{~B}_{n}$ of a specified geometry (shape) and number of atoms $\mathrm{m}+\mathrm{n}$ compared to the corresponding monometallic particles $\mathrm{A}_{m+n}$ and $\mathrm{B}_{m+n}$ : (1) the composition $-m: n$ ratio and (2) the atomic ordering - pattern of positions that atoms A and B occupy in this particle geometry. Structures different only by positions of atoms A and B in a given particle geometry, denoted as homotops [18], rapidly increase in number with particle size. For instance, already for $\sim 1 \mathrm{~nm}$ large $\mathrm{A}_{40} \mathrm{~B}_{40}$ particle of just 80 atoms the number of homotops (including symmetry-equivalent ones) reaches an astronomical value of $10^{23}$. This limits comprehensive exploration of the homotop landscape to much smaller particles than those consisting of many hundred to several thousand atoms common in catalytic applications.

State-of-the-art modelling atomic ordering of alloy particles combines DFT calculations [19] with cluster expansion method [20]. Extension of this sophisticated approach to treat bimetallic particles with adsorbates allowed Wang et al. [21] to comparatively study bare and oxygen-covered cuboctahedral 55-atomic $\mathrm{Pd}-\mathrm{Rh}$ particles at various $\mathrm{Pd}: \mathrm{Rh}$ compositions. Bare NPs were shown to exhibit a simple alloying behavior and strong Rh-core/Pd-shell preference. Gradual increase of oxygen coverage caused $\mathrm{Rh}$ atoms to emerge on the surface, up to a total reversal to Pd-core/Rh-shell. Yet, the studied 55-atomic $\mathrm{Pd}-\mathrm{Rh}$ particles with very high ratio of surface to inner atoms may not be in the scalable-with-size regime [22-24] required to represent in sufficient details surface structures and segregation of atoms in Pd-Rh NPs of much larger sizes typical for technical catalysts. In order to address this issue, we modelled in the present work $\mathrm{Pd}-\mathrm{Rh}$ NPs of different $\mathrm{Pd}: \mathrm{Rh}$ ratios formed of 140 to 3630 atoms ( 1.4 to $5.4 \mathrm{~nm}$, respectively) using a novel efficient tool for optimising atomic ordering of large bimetallic NPs $[25,26]$.

Among the main goals of our study are: (1) to quantify using DFT calculations atomic ordering and segregation effects in Pd-Rh NPs containing up to 201 atoms at varying Pd : Rh compositions; (2) using these data to describe with DFT accuracy energetically preferred atomic orderings in inaccessible by DFT larger Pd-Rh NPs with sizes 4-5 nm common for catalytic metal particles; (3) to illustrate how one can evaluate induced by adsorbates expected segregation effects on the surface arrangement of Pd-Rh nanoalloy catalysts.

\section{Computational details}

DFT calculations were performed with the help of a plane-wave code VASP $[27,28]$. A gradient-corrected Perdew-Becke-Ernzerhof (PBE) exchange-correlation functional [29] was employed in combination with the projector augmented wave representation of core electrons [30,31]. Only $\Gamma$-point was used for the Brillouin zone sampling. Cutoff energy for the wave functions was $250.925 \mathrm{eV}$. One-electron levels were smeared by $0.1 \mathrm{eV}$ and the converged energies were finally extrapolated to the zero smearing. All atoms were locally relaxed without any restrictions until forces on each atom became less than $0.02 \mathrm{eV}$.

Our common DFT models of Pd-Rh nanoalloy particles with varying $\mathrm{Pd}: \mathrm{Rh}$ composition comprised 201 atoms. These truncated octahedral NPs with $f c c$ structure were located in $2.5 \times 2.5 \times 2.5 \mathrm{~nm}$ large periodically repeated cells with the $\geq 0.9$ $\mathrm{nm}$ separation between adjacent particles. Interactions of metal NPs at such distances are shown to be negligible [32].

We determined contributions governing the atomic arrangement (atomic or chemical ordering) in Pd-Rh nanoalloys employing our recent method [25] for the global optimization of the ordering in a NP of a given shape, size and composition. As described in details elsewhere $[25,26]$, this method, hereafter denoted TOP, is based on simple energy expressions defined by locations of atoms of two metals in crystalline positions of the NP, i.e. solely by the topology of the latter. The TOP method is widely applicable to bimetallic nanocrystallites composed of different metals. Deviation from the crystallinity, e.g. in small, sub-nanometer particles or in combinations of metals with large mismatch of the atomic sizes, is one of few factors limiting the applicability. The method involves the use of $\varepsilon_{i}$ parameters (descriptors) associated either with the surface segregation energy of Pd atoms or the interaction energy of Pd-Rh pairs of the nearest atoms (denoted hereafter Pd-Rh bond energy, which can also be destabilising). The values of $\varepsilon_{i}$ were obtained via fitting to DFT energies of several dozens of NP structures with different chemical orderings. The resulting TOP expressions were employed in efficient Monte-Carlo (MC) simulations to find globally optimised atomic orderings in Pd-Rh NPs with the accuracy of DFT calculations.

\section{Results and discussion}

\subsection{Atomic ordering in $P d_{70} R h_{70}, P d_{50} R h_{151}, P d_{101} R h_{100}$ and $P d_{151} R h_{50}$ nanoparticles from DFT calculations}

Our DFT results calculated for two types of truncated octahedral model Pd-Rh NPs with different Pd : Rh compositions, $\mathrm{Pd}_{50} \mathrm{Rh}_{151}$ (1:3), $\mathrm{Pd}_{101} \mathrm{Rh}_{100}$ and $\mathrm{Pd}_{70} \mathrm{Rh}_{70}(1: 1)$ and $\mathrm{Pd}_{151} \mathrm{Rh}_{50}$ ( $3: 1)$, are presented in Figs. 1 and 2 in the form of structural sketches corresponding to the lowest-energy optimised atomic ordering for each kind of the NPs. Pre-screening of various atomic orderings for finding putative lowest-energy homotops was performed by MC simulations [25] using the TOP energy descriptors $\varepsilon_{i}$ listed in Table 1 . The descriptors define TOP expressions for energy difference $\Delta E$ TOP between any two homotops of a given Pd-Rh NP.

$$
\begin{gathered}
\Delta E_{\mathrm{TOP}}=\varepsilon_{B O N D}^{P d-R h} \Delta N_{B O N D}^{P d-R h}+\varepsilon_{C O R E R}^{P d} \Delta N_{C O R N E R}^{P d}+\varepsilon_{E D G E}^{P d} \Delta N_{E D G E}^{P d}+ \\
\varepsilon_{T E R R A C E}^{P d} \Delta N_{T E R R A C E}^{P d}
\end{gathered}
$$

which depends on the number of Pd-Rh bonds $\left(N_{B O N D}^{P d-R h}\right)$ and the numbers of corner $\left(N_{C O R N E R}^{P d}\right)$, edge $\left(N_{E D G E}^{P d}\right)$ and terrace $\left(N_{T E R R A C E}^{P d}\right)$ surface Pd atoms in each homotop.

The following individual TOP expressions (in eV) correspond to four kinds of the calculated by DFT Pd-Rh NPs with 140 and 201 atoms: 

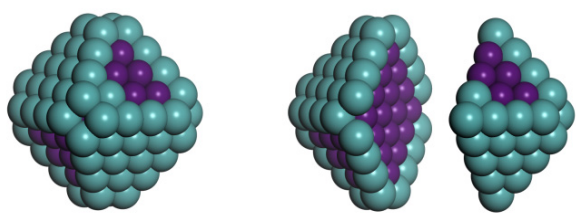

Fig. 1. Atomic ordering in the lowest-energy homotop of $1.4 \mathrm{~nm}$ large truncated octahedral $\mathrm{Pd}_{70} \mathrm{Rh}_{70}$ particle. As shown in the split image of the particle in the right panel, its inner region consists of $\mathrm{Rh}$ atoms only. Color coding: Pd - turquoise, $\mathrm{Rh}$ - purple.

$$
\begin{gathered}
\Delta E_{\mathrm{TOP}}\left(\mathrm{Pd}_{70} \mathrm{Rh}_{70}\right)=0.021 \Delta N_{B O N D}^{P d-R h}-0.814 \Delta N_{C O R N E R}^{P d} \\
-0.588 \Delta N_{E D G E}^{P d}-0.456 \Delta N_{T E R R A C E}^{P d} \\
\Delta E_{\mathrm{TOP}}\left(\mathrm{Pd}_{50} \mathrm{Rh}_{151}\right)=0.018 \Delta N_{B O N D}^{P d-R h}-0.603 \Delta N_{C O R N E R}^{P d} \\
-0.631 \Delta N_{E D G E}^{P d}-0.544 \Delta N_{T E R R A C E}^{P d} \\
\Delta E_{\mathrm{TOP}}\left(\mathrm{Pd}_{101} \mathrm{Rh}_{100}\right)=0.024 \Delta N_{B O N D}^{P d-R h}-0.755 \\
\Delta N_{\text {CORNER }}^{P d}-0.536 \Delta N_{E D G E}^{P d}-0.356 \Delta N_{T E R R A C E}^{P d} \\
\Delta E_{\mathrm{TOP}}\left(\mathrm{Pd}_{151} \mathrm{Rh}_{50}\right)=0.032 \Delta N_{B O N D}^{P d}-0.691 \Delta N_{C O R N E R}^{P d} \\
-0.597 \Delta N_{E D G E}^{P d}-0.362 \Delta N_{T E R R A C E}^{P d}
\end{gathered}
$$

\subsubsection{Pd70Rh70 nanoparticle}

Fig. 1 displays the lowest-energy homotop of the $\mathrm{Pd}_{70} \mathrm{Rh}_{70}$ NP. Pd forms there a part of the 96-atomic monolayer thick shell, in which all $70 \mathrm{Pd}$ atoms are located. They occupy all 48 available corner and edge positions along with 22 of 48 available positions on the $\{111\}$ terraces. The remaining 26 surface terrace sites of the NP are occupied by compact nano-islands of $\mathrm{Rh}$ atoms. The inner part (core) of the NP completely consists of $44 \mathrm{Rh}$ atoms. These results illustrate propensity of $\mathrm{Pd}$ atoms to be strongly energetically stabilised in low-coordinated surface sites of Pd-Rh particles. Indeed, the TOP descriptor values (Table 1) estimate the energy gain to be $814 \mathrm{meV}$ for each Pd atom emerging from the NP core in a 6-coordinated corner position, assuming unchanged total number of nearest-neighbor Pd-Rh contacts (bonds). The corresponding energy gains are 588 and $456 \mathrm{meV}$ for the appearance of a core $\mathrm{Pd}$ atom in 7-coordinated edge and 9-coordinated $\{111\}$ terrace positions, respectively. This revealed by our DFT calculations behavior of Pd to surface segregation in nanoalloys with $\mathrm{Rh}$ (atoms of which are only $3 \mathrm{pm}$ smaller than Pd ones) is fully consistent with the already mentioned for extended systems lower surface energy of Pd compared with Rh [15] and a clear preference of $\mathrm{Pd}(\mathrm{Rh})$ atom impurities in $\mathrm{Rh}(\mathrm{Pd})$ slabs to surface (inner) locations [16].

Data in Table 1 for the $\mathrm{Pd}_{70} \mathrm{Rh}_{70}$ NP point to destabilising interaction between the nearest $\mathrm{Pd}$ and $\mathrm{Rh}$ atoms (Pd-Rh bond) vs. a half of the sum of two monometallic Pd-Pd and Rh-Rh bonds, by $21 \mathrm{meV}$ on average. Hence, the immiscibility of Pd and $\mathrm{Rh}$ in the bulk (vide supra) still noticeably affects alloying propensity of these metals in $1.5 \mathrm{~nm}$ large $1: 1 \mathrm{Pd}$ :Rh NPs.

Next we explore how the findings for the $\mathrm{Pd}_{70} \mathrm{Rh}_{70} \mathrm{NP}$ depend on the size and composition based on our DFT data for larger 201-atomic Pd-Rh NPs with varying Pd : Rh contents.

\subsubsection{1 atomic $P d$-Rh nanoparticles with $1: 3,1: 1$ and $3: 1$ compositions}

Pd101Rh10o nanoparticle. Results for larger $1: 1$ Pd-Rh NP

$\mathrm{Pd}_{101} \mathrm{Rh}_{100}$ (Table 1 and Fig. 2) are similar to those for $\mathrm{Pd}_{70} \mathrm{Rh}_{70}$ NP. Again, Pd atoms are notably stabilised on the surface with the stabilisation magnitude correlating with the coordination numbers of the surface sites. The stabilisation ranges from 755 $\mathrm{meV}$ in corner sites with the lowest coordination to $356 \mathrm{meV}$ in $\{111\}$ terrace sites with the highest surface coordination. Notably, these descriptors characterising surface segregation energy of $\mathrm{Pd}$ atoms in the $\mathrm{Pd}_{101} \mathrm{Rh}_{100} \mathrm{NP}$ practically coincide (with the statistical accuracy) with the corresponding descriptors for the $\mathrm{Pd}_{70} \mathrm{Rh}_{70} \mathrm{NP}$ and the Pd-Rh bond energy descriptors are also statistically indistinguishable for the both NPs with the same $1: 1 \mathrm{Pd}: \mathrm{Rh}$ composition. Hence, it is not surprising that the atomic ordering in the $\mathrm{Pd}_{101} \mathrm{Rh}_{100} \mathrm{NP}$ (Fig. 2) essentially does not alter with the particle size increase from $\mathrm{Pd}_{70} \mathrm{Rh}_{70}$. As in the latter, $\mathrm{Pd}$ atoms form a monatomic shell in the $\mathrm{Pd}_{101} \mathrm{Rh}_{100}$ NP, which is not complete solely because of the insufficient number of $101 \mathrm{Pd}$ atoms to occupy all exposed surface sites. These observations indicate that the general structural and related features of quite small $\mathrm{Pd}_{70} \mathrm{Rh}_{70}$ and $\mathrm{Pd}_{101} \mathrm{Rh}_{100} \mathrm{NPs}$ should be representative of the corresponding features of notably larger Pd-Rh NPs with the same composition, in line with results for other bimetallic NPs of similar sizes [25,26,33,34]. But what about atomic ordering in Pd-Rh NPs with other compositions different from $1: 1$ ?

$\boldsymbol{P d}_{50} \boldsymbol{R h}_{151}$ nanoparticle. The surface segregation propensity of Pd atoms in 201-atomic Pd-Rh NP at smaller Pd content remains substantial. Yet, the noticed for $1: 1 \mathrm{Pd}-\mathrm{Rh}$ NPs clear dependence of the segregation energy on the coordination numbers of particular surface sites becomes less expressed in the $\mathrm{Pd}_{50} \mathrm{Rh}_{151} \mathrm{NP}$ (Table 1). There, the segregation of Pd to

\begin{tabular}{|c|c|c|c|}
\hline & $\mathrm{Pd}_{50} \mathrm{Rh}_{151}$ & $\begin{array}{c}\mathrm{Pd}_{101} \mathrm{Rh}_{100} \\
\mathrm{Pd}_{70} \mathrm{Rh}_{70} \\
\end{array}$ & $\mathrm{Pd}_{151} \mathrm{Rh}_{50}$ \\
\hline \multirow[t]{2}{*}{$\varepsilon_{B O N D}^{P d-R h}$} & $18_{-21}^{+14}$ & $24_{-6}^{+6}$ & $32_{-7}^{+6}$ \\
\hline & & $21_{-2}^{+2}$ & \\
\hline \multirow[t]{2}{*}{$\varepsilon_{\text {CORNER }}^{P d}$} & $-603_{-87}^{+60}$ & $-755_{-83}^{+101}$ & $-691_{-95}^{+91}$ \\
\hline & & $-814_{-23}^{+34}$ & \\
\hline \multirow[t]{2}{*}{$\varepsilon_{E D G E}^{P d}$} & $-631_{-152}^{+107}$ & $-536_{-47}^{+40}$ & $-597_{-46}^{+39}$ \\
\hline & & $-588_{-37}^{+26}$ & \\
\hline \multirow[t]{2}{*}{$\varepsilon_{T E R R A C E}^{P d}$} & $-544_{-75}^{+87}$ & $-356_{-40}^{+41}$ & $-362_{-14}^{+16}$ \\
\hline & & $-456_{-46}^{+30}$ & \\
\hline \multirow[t]{2}{*}{$N_{\text {FIT }}$} & 30 & 31 & 29 \\
\hline & & 44 & \\
\hline \multirow[t]{2}{*}{ precision, $\delta\left(E_{\mathrm{DFT}}-E_{\mathrm{TOP}}\right)$} & 131 & 348 & 127 \\
\hline & & 519 & \\
\hline \multirow[t]{2}{*}{ accuracy, $\Delta E$} & 0 & 123 & 0 \\
\hline & & 125 & \\
\hline
\end{tabular}
6-coordinated corner sites is not the most energetically pref-

\section{Table 1}

Descriptors $\varepsilon_{i}{ }^{\mathrm{a}}$ in the topological energy expressions $\Delta E_{\mathrm{TOP}}{ }^{\mathrm{b}}$ for Pd-Rh nanoparticles of 1:3 $\left(\mathrm{Pd}_{50} \mathrm{Rh}_{151}\right), 1: 1\left(\mathrm{Pd}_{70} \mathrm{Rh}_{70}, \mathrm{Pd}_{101} \mathrm{Rh}_{100}\right)$ and $3: 1$ $\left(\mathrm{Pd}_{151} \mathrm{Rh}_{50}\right)$ compositions along with their precision $\delta$ and accuracy $\Delta E$, also showing number of DFT-optimised structures NFIT used for defining the TOP expressions. All energies are in meV. 


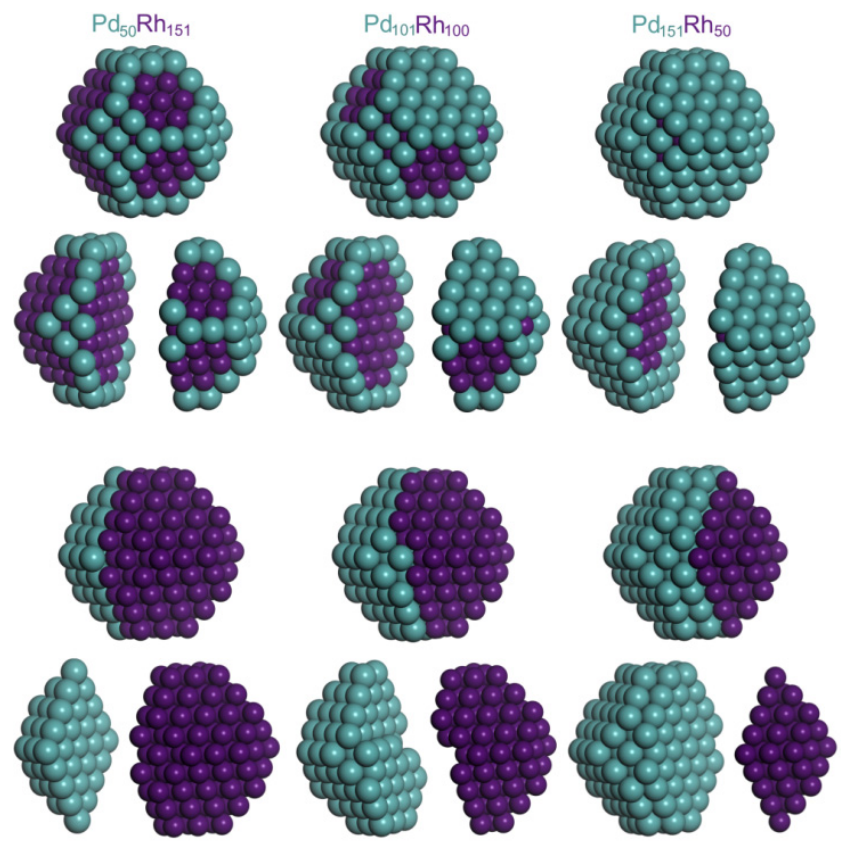

Fig. 2. Atomic ordering in the lowest-energy (upper two rows) and Janus-type (lower two rows) homotops of $1.7 \mathrm{~nm}$ large truncated octahedral 201-atomic NPs with varying Pd:Rh composition: 1:3 $\mathrm{Pd}_{50} \mathrm{Rh}_{151}, 1: 1-\mathrm{Pd}_{101} \mathrm{Rh}_{100}$ and 3:1 $-\mathrm{Pd}_{151} \mathrm{Rh}_{50}$. Color coding as in Fig. 1.

erable anymore and 7-coordinated edge sites turn out to be similarly stabilising for Pd atoms. The lowest-energy atomic ordering $\mathrm{Pd}_{50} \mathrm{Rh}_{151}$ homotop (Fig. 2) exhibits all $50 \mathrm{Pd}$ atoms located solely in the corner (19), edge (27) and $\{100\}$ terrace (4) sites, with the shell completed by $72 \mathrm{Rh}$ atoms. The core is formed of only Rh atoms, Rh79. No statistically significant alteration of the energetics of pair Pd-Rh interactions is observed.

$\boldsymbol{P d}_{151} \boldsymbol{R h}_{50}$ nanoparticle. No important differences in segregation and bonding properties of $\mathrm{Pd}$ and $\mathrm{Rh}$ atoms are calculated for 3 : 1 Pd-Rh NP Pd $\mathrm{d}_{151} \mathrm{Rh}_{50}$ (Table 1, Fig. 2). Due to higher Pd content, there are sufficient atoms to form a purely Pd shell in it. A 79-atomic core of $\mathrm{Pd}_{151} \mathrm{Rh}_{50}$ consists of $50 \mathrm{Rh}$ and $29 \mathrm{Pd}$ atoms. The clear trend of more stabilised Pd atoms in less coordinated surface positions is the same as for the $1: 1$ $\mathrm{Pd}-\mathrm{Rh}$ NPs addressed above. Pd-Rh interactions are calculated slightly more destabilising than for the other compositions under scrutiny. Notably, core-shell atomic ordering of Pd and $\mathrm{Rh}$ components remains characteristic for the studied 201-atomic Pd-Rh NPs at all compositions. This deserves a closer look in view of the known immiscibility of Pd and Rh in bulk [11-14].

\subsubsection{On the mixing of $P d$ and $R h$ components}

One way to analyse and quantify miscibility of Pd and Rh at the nanoscale is to compare stability of the lowest-energy Rh-core/Pd-shell homotops (Fig. 2, upper panels) with stability of the homotops featuring as much as possible separated components of these two metals. As such models of most separated components of the 201-atomic Pd-Rh NPs we have chosen homotops with the so-called Janus-type atomic ordering (Fig. 2, lower panels). Number of the nearest-neighbour Pd-Rh pairs, which destabilise mixing of these two metals according to the positive Pd-Rh bond energy descriptors $\varepsilon_{B O N D}^{P d-R h}$ (Table 1), is in the Janus-type structures only a half of that in the corresponding core-shell structures: 96 vs. 197 for $\mathrm{Pd}_{50} \mathrm{Rh}_{151}, 128$ vs. 270 for $\mathrm{Pd}_{101} \mathrm{Rh}_{100}$, and 96 vs. 208 for $\mathrm{Pd}_{151} \mathrm{Rh}_{50}$. Nevertheless, DFT (TOP) energies of the Janus structures are higher than those of the respective optimised lowest-energy structures by as much as (in eV): 9.68 (7.56) - $\mathrm{Pd}_{50} \mathrm{Rh}_{151}, 18.21$ (18.79) - $\mathrm{Pd}_{101} \mathrm{Rh}_{100}$ and 14.47 (14.32) - $\mathrm{Pd}_{151} \mathrm{Rh}_{50}$. Such overcoming of the destabilising effect of the Pd-Rh contacts in the bimetallic particles is related to a very substantial energy gain due to strong propensity of Pd atoms to segregate on the surface forming a shell around a core of $\mathrm{Rh}$. We note here in passing that the good agreement of the DFT and TOP relative energies of the NPs with Janus and core-shell orderings corroborates applicability of the TOP expressions to estimate (almost with DFT accuracy) stabilities of various homotops, both low-lying and quite high in energy.

One can also evaluate miscibility of $\mathrm{Pd}$ and $\mathrm{Rh}$ at the nanoscale by calculating so-called excess energy of Pd-Rn NPs versus monometallic Pd and Rh NPs with the same numbers of atoms and structure. For the studied $\mathrm{Pd}_{201-n} \mathrm{Rh}_{n} \mathrm{NPs}$ the excess energy per atom $[35,36]$ is:

$$
\begin{gathered}
E_{\text {exc }}\left(\mathrm{Pd}_{201-n} \mathrm{Rh}_{n}\right)=\left\{E\left(\mathrm{Pd}_{201-n} \mathrm{Rh}_{n}\right)-\right. \\
\left.[(201-n) / 201] E\left(\mathrm{Pd}_{201}\right)-(n / 201) E\left(\mathrm{Rh}_{201}\right)\right\} / 201
\end{gathered}
$$

The negative $E_{\text {exc }}$ values (Fig. 3 ) indicate miscibility, in full agreement with our observation of the preference of $\mathrm{Rh}$-core/Pd-shell atomic orderings over Janus-type ones with more separate $\mathrm{Pd}$ and $\mathrm{Rh}$ components. Interestingly, the $E_{\mathrm{exc}}$ values of $0.06-0.09 \mathrm{eV}$ per atom corresponding to the $\mathrm{Pd}: \mathrm{Rh}$ ratios 1:3, 1:1 and 3:1 are close to the values calculated for nanoalloys with such well miscible atoms as Pd-Au [11-14].

\subsection{Atomic ordering in bare Pd-Rh nanoparticles containing thousands atoms}

Using TOP descriptors from Table 1 determined for 140and 201-atomic Pd-Rh NPs and defined by them TOP Eqs. (2) (5) we performed a MC search for the lowest-energy homotops of truncated octahedral and cuboctahedral Pd-Rh NPs formed

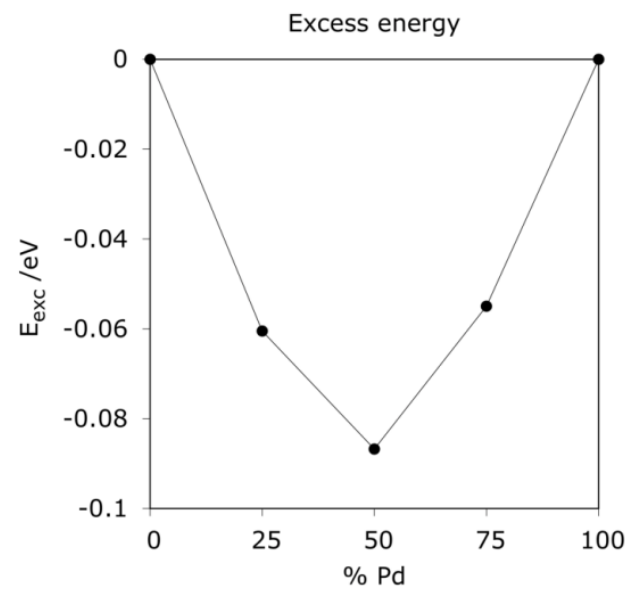

Fig. 3. DFT excess energies $E_{\text {exc }}$ per atom of $\operatorname{Pd}_{201-n} \mathrm{Rh}_{n}(n=50,100,151)$ NPs. 
of 1463 and 3630 atoms, respectively. The sizes of these NPs, ca. 4.4 and $5.4 \mathrm{~nm}$, relevant for catalytic applications are well beyond sizes accessible by ordinary DFT calculations. The Pd : Rh compositions $1: 3,1: 1$ and $3: 1$ have been studied for both 1463- (Fig. 4) and 3630-atomic (Fig. 5) NPs.

In all cases the lowest-energy homotops of these NPs exhibit core-shell structures with the shells consisting of Pd atoms and the cores formed of $\mathrm{Rh}$ atoms. Hence, the atomic ordering patterns of quite large NPs with somewhat different shapes are qualitatively very similar to the patterns of the NPs smaller than $2 \mathrm{~nm}$. The main effect causing some differences in the surface compositions of the smaller and larger NPs at a given $\mathrm{Pd}: \mathrm{Rh}$ content is the ratio of surface (corner + edge + terrace) to inner atoms, which rapidly decreases with increasing particle size. This ratio $(24+36+62): 79=1.54$ for the 201 -atomic NPs drops to $(24+108+440): 891=0.64$ for the 1463 -atomic $\mathrm{NPs}$ and to just $(24+192+744): 2670=0.36$ for the 3630-atomic NPs.

What justifies application of the TOP descriptors and expressions determined for the 140- and 201-atomic NPs to model realistically enough atomic ordering in much larger NPs? The evidence for that discussed in the TOP studies of other bimetallic NPs $[25,26,33,34]$ is that TOP descriptors calculated for ca. 100-atomic particles change only insignificantly for still accessible by DFT larger NPs having the same proportion of two metals. This is also the case for the Pd-Rh NPs under scrutiny, as revealed (vide supra) by very close resemblance of the TOP descriptors for $1: 1$ NPs of two sizes, $\mathrm{Pd}_{70} \mathrm{Rh}_{70}$ and $\mathrm{Pd}_{101} \mathrm{Rh}_{100}$ (Table 1). This resemblance is evidenced by images in the central columns of Fig. 4 (for $\mathrm{Pd}_{731} \mathrm{Rh}_{732}$ ) and of Fig. 5 (for $\mathrm{Pd}_{1815} \mathrm{Rh}_{1815}$ ). The upper images sketch atomic ordering of the lowest-energy homotops optimised using the TOP descriptors obtained for the larger $\mathrm{Pd}_{101} \mathrm{Rh}_{100} \mathrm{NP}$, whereas the lower images with very similar orderings correspond to the lowest-energy homotops optimised using the TOP descriptors for $\mathrm{Pd}_{70} \mathrm{Rh}_{70}$. The TOP energy differences between these differently optimised pairs of $\mathrm{Pd}_{731} \mathrm{Rh}_{732}$ and $\mathrm{Pd}_{1815} \mathrm{Rh}_{1815}$ homotops are negligibly small, only $0.14 \mathrm{eV}\left(\Delta N_{B O N D}^{P d-R h}=6\right)$ and $0.29 \mathrm{eV}$ $\left(\triangle N_{B O N D}^{P d-R h}=12\right)$, respectively. Applicability of the TOP de-

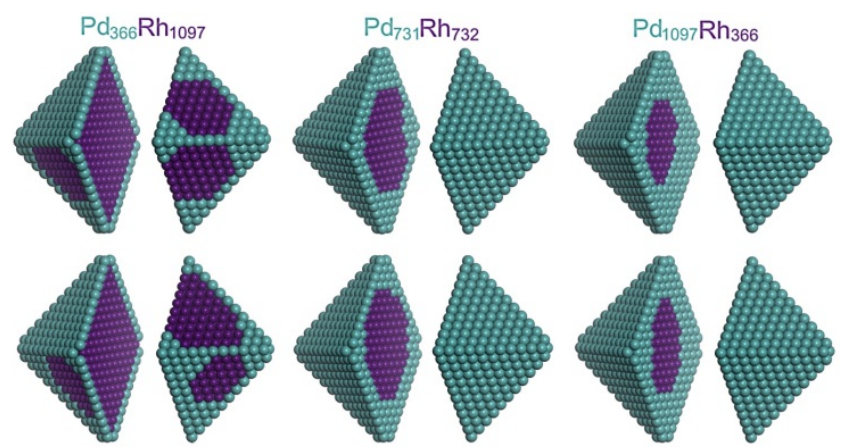

Fig. 4. Atomic ordering in the lowest-energy homotops of ca. $4.4 \mathrm{~nm}$ large truncated octahedral 1463-atomic NPs with varying $\mathrm{Pd}: \mathrm{Rh}$ composition: 1:3 - $\mathrm{Pd}_{366} \mathrm{Rh}_{1097}, 1: 1-\mathrm{Pd}_{731} \mathrm{Rh}_{732}$ and 3:1 $-\mathrm{Pd}_{1097} \mathrm{Rh}_{366}$. Upper panels (from left to right) display homotops calculated using the TOP Eqs. (3), (4) and (5), respectively. Images in the corresponding lower panels are obtained using the equations 4,2 and 4 . Color coding is the same as in Fig. 1.

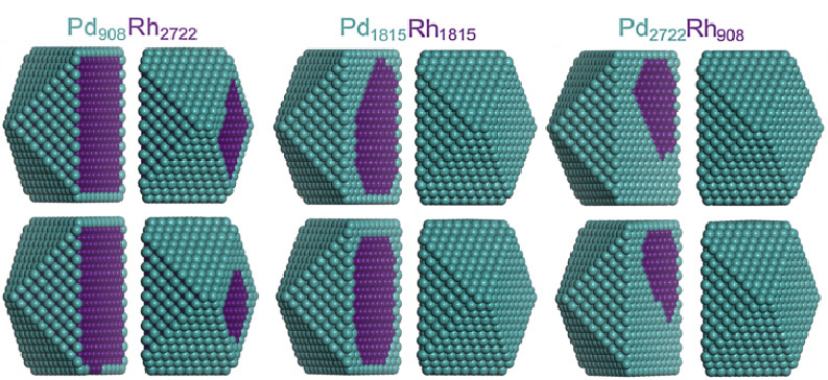

Fig. 5. Atomic ordering in the lowest-energy homotops of ca. $5.4 \mathrm{~nm}$ large cube-octahedral 3630-atomic NPs with varying Pd : Rh composition: 1:3 - $\mathrm{Pd}_{908} \mathrm{Rh}_{2722}$, 1:1 $-\mathrm{Pd}_{1815} \mathrm{Rh}_{1815}$ and 3:1 - $\mathrm{Pd}_{2722} \mathrm{Rh}_{908}$. Upper panels (from left to right) display homotops calculated using the TOP Eqs. (3), (4) and (5), respectively. Images in the corresponding lower panels are obtained using the Eqs. (4), (2) and (4). Color coding is the same as in Fig. 1.

scriptors to notably larger Pd-Rh NPs is also supported by another important finding, which was not made previously for bimetallic NPs [25,26,33,34]. Namely, that very similar ordering patterns of the lowest-energy homotops result from the application of the TOP descriptors for both the same and different $\mathrm{Pd}: \mathrm{Rh}$ ratios. One can see, for instance, qualitatively very similar orderings in the upper and lower images of $1: 3$ $\mathrm{Pd}_{366} \mathrm{Rh}_{1097} \mathrm{NP}$ in Fig. 4 optimised using TOP descriptors for NPs with the same ratio $1: 3\left(\mathrm{Pd}_{50} \mathrm{Rh}_{151}\right)$ and with different ratio 1 : $1\left(\mathrm{Pd}_{101} \mathrm{Rh}_{100}\right)$, respectively. The same conclusion could be drawn for the other pairs of homotops $\mathrm{Pd}_{1097} \mathrm{Rh}_{366}$, $\mathrm{Pd}_{908} \mathrm{Rh}_{2722}$ and $\mathrm{Pd}_{2722} \mathrm{Rh}_{908}$ shown in Figs. 4 and 5.

DFT and TOP data presented so far correspond to $0 \mathrm{~K}$. The TOP method allows estimating properties associated with the Boltzmann population of different homotops of a particular NP at a given temperature by accounting for entropy contributions related with atomic ordering, not considering atomic vibrations [26]. We applied this protocol to calculate probabilities of occupying each site by either Pd or Rh atoms and to estimate average atomic orderings in the 1463-atomic NPs with Pd: Rh ratios $1: 3$ and $1: 1$ at $600 \mathrm{~K}$ and $1000 \mathrm{~K}$ (Fig. 6 and Table 2).

Temperature increase mainly acts on $\mathrm{Pd}_{366} \mathrm{Rh}_{1097} \mathrm{NP}$ to exchange Pd atoms, all of which are on the surface, with surface $\mathrm{Rh}$ atoms. It transforms compact surface $\mathrm{Rh}$ islands present at $0 \mathrm{~K}$ into smaller and less regular ones at $600 \mathrm{~K}$ and causes
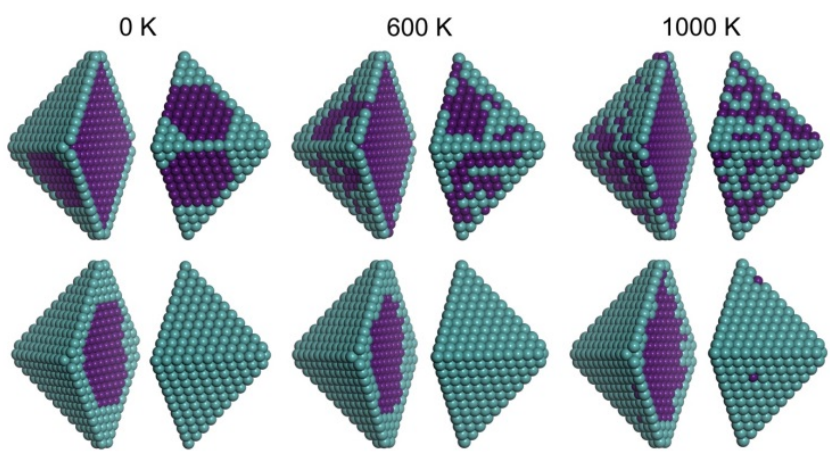

Fig. 6. Temperature effects on the atomic ordering in the representative homotops of truncated octahedral 1463-atomic Pd-Rh NPs at $0 \mathrm{~K}$, $600 \mathrm{~K}$ and $1000 \mathrm{~K}$. Upper panels - $\mathrm{Pd}_{366} \mathrm{Rh}_{1097}$, bottom panels $\mathrm{Pd}_{731} \mathrm{Rh}_{732}$. Color coding is the same as in Fig. 1. 
Table 2

Temperature dependence of the TOP atomic ordering in the representative homotops of truncated octahedral $\mathrm{Pd}_{366} \mathrm{Rh}_{1097}$ and $\mathrm{Pd}_{731} \mathrm{Rh}_{732}$ nanoparticles shown in Fig. 6.

\begin{tabular}{lccc}
\hline System & \multicolumn{3}{c}{ Temperature, $\mathrm{K}$} \\
\cline { 2 - 4 } $\mathrm{Pd}_{366} \mathrm{Rh}_{1097}$ & 0 & 600 & 1000 \\
$N_{B O N D}^{P d-R h}$ & 1060 & 1419 & 1555 \\
$N_{C O R N E R}^{P d}$ & 24 & 23 & 20 \\
$N_{E D G E}^{P d}$ & 108 & 103 & 93 \\
$N_{T E R R A C E}^{P d}$ & 234 & 240 & 252 \\
$\mathrm{Pd}_{731} \mathrm{Rh} \mathrm{h}_{732}$ & & & \\
$N_{B O N D}^{P d-R h}$ & 1238 & 1331 & 1766 \\
$N_{C O R N E R}^{P d}$ & 24 & 24 & 24 \\
$N_{E D G E}^{P d}$ & 108 & 108 & 107 \\
$N_{T E R R A C E}^{P d}$ & 440 & 440 & 435 \\
\hline
\end{tabular}

$N_{B O N D}^{P d-R h}$ - number of Pd-Rh bonds (nearest neighbours);

$N_{\text {CORNER }}^{P d}, N_{E D G E}^{P d}$ and $N_{T E R R A C E}^{P d}$ - numbers of Pd surface atoms in the corner, edge and terrace positions, respectively.

complete disappearance of the islands of $\mathrm{Rh}$ at $1000 \mathrm{~K}$. This enhanced mixing is manifested by substantially increased number of Pd-Rh pairs of atoms and more terrace Pd atoms formed by displacements from corner and edge sites (Table 2). Interestingly, the propensity of $\mathrm{Pd}$ atoms to remain on the surface in Pd-Rh nanoalloys revealed by the TOP descriptors (Table 1) is so strong that almost no temperature-induced exchange of surface Pd atoms with inner Rh atoms has been calculated. Even at $1000 \mathrm{~K}$ the representative homotop of the $\mathrm{Pd}_{366} \mathrm{Rh}_{1097} \mathrm{NP}$ is predicted to exhibit just one inner Pd atom (Table 2). $\mathrm{Pd}_{731} \mathrm{Rh}_{732} \mathrm{NP}$ featuring at $0 \mathrm{~K}$ a compact Rh core covered by a Pd shell thicker than one atomic layer exhibits even weaker temperature effects (see bottom images in Fig. 6 and Table 2) than $\mathrm{Pd}_{366} \mathrm{Rh}_{1097} \mathrm{NP}$. Only at $1000 \mathrm{~K}$ emergence of few single $\mathrm{Rh}$ atoms on the surface of $\mathrm{Pd}_{731} \mathrm{Rh}_{732} \mathrm{NPs}$ becomes energetically feasible. Such small influence of temperature on the atomic ordering of Pd-Rh NPs compared, for instance with that of Pd-Au NPs [26], is related with both the immiscibility of $\mathrm{Pd}$ and $\mathrm{Rh}$ atoms and quite strong preference of $\mathrm{Pd}$ atoms to stay on the surface. Notably, the simple TOP descriptors allow rationalising and predicting atomic ordering differences in the surface segregation of different nanoalloys and their temperature dependence.

\subsection{On the reactive media effects on the surface arrangement of catalytic nanoparticles}

As mentioned in the Introduction, atomic orderings of Pd-Rh NPs under experimental conditions can be very different from the Pd-shell/Rh-core arrangement predicted by our modelling as energetically the most stable in vacuum. Provided that experimentally prepared catalytic Pd-Rh NPs were annealed long enough at sufficiently high temperature to minimise the internal energy, the major effects triggering their atomic ordering to deviate from Pd-shell/Rh-core are usually interactions of the NPs with adsorbates and supports $[7,10]$.

There are several levels of the computational modelling ap- proaches to address surface restructuring of bimetallic NPs caused by interactions with adsorbates present in reactive environments. A rigorous approach, albeit too computationally intensive for routine applications to particles larger than $1 \mathrm{~nm}$, is an extension of the cluster expansion method to DFT treatment of bimetallic particles with adsorbates [21]. The TOP approach used in the present work was also employed to evaluate surface segregation effects induced by adsorbates in quite large bimetallic NPs [34,37]. The latter predictions of the surface ordering relied on a simple concept that an adsorbate more strongly interacting with surface sites formed by atoms of metal one than by atoms of metal two energetically stabilises surface atoms of the metal one and the degree of such stabilisation is defined by the adsorption energy difference for the most strongly binding sites of these metals forming the studied nanoalloy. In this way, DFT calculations of $\mathrm{CH}_{x}$ adsorbates on $\mathrm{Cu}$ and Ni sites of $\mathrm{Cu}-\mathrm{Ni}$ NPs in combination with the TOP descriptors defining surface segregation in the bare NPs quantified coverages of the $\mathrm{CH}_{x}$ adsorbates required to cover under reaction conditions $\mathrm{Cu}-\mathrm{Ni} \mathrm{NPs}$ by active $\mathrm{Ni}$ atoms instead of inert $\mathrm{Cu}$ ones, as was desired for improving the catalytic function [34]. Similarly, employment of TOP descriptors together with DFT adsorption energies of CO molecules on Pd and $\mathrm{Au}$ sites of Pd-Au NPs enabled predicting CO coverages, at which experimentally observed significant $\mathrm{CO}$-induced surface segregation of Pd takes place [37].

In case of well-ordered metal NPs with abundant sites at extended terraces that bind adsorbates similarly strong as the corresponding single-crystal surfaces [22] one can greatly reduce computational expenditures employing adsorption preference energy defined as a difference of binding energies of a given adsorbate on surfaces of two metals forming bimetallic NPs under study [38]. If less quantitative predictions of the adsorbate-induced surface segregation are sufficient, one can merely use published adsorption energies of the most relevant reactants and intermediates on single-crystal monometallic surfaces and thus to completely avoid expensive DFT calculations of NPs with adsorbates.

To shed light on induced by adsorbates changes of the surface atomic ordering in Pd-Rh NPs we calculated adsorption energies of $\mathrm{O}$ atom as well as $\mathrm{CO}$ and NO molecules on 6-layers thick $\mathrm{Pd}(111)$ and $\mathrm{Rh}(111)$ slabs with $3 \times 3$ surface cells. The following DFT (PBE) adsorption energies (in eV) are obtained on $\mathrm{Pd}(111)$ and $\mathrm{Rh}$ (111), respectively: $\mathrm{O}-4.64$ and 5.22, CO 2.16 and 1.99, NO -2.37 and 2.49. From these data the adsorption preference of $\mathrm{O}$ to the $\mathrm{Rh}$ surface $v s$. $\mathrm{Pd}$ one is $0.58 \mathrm{eV}$ and that of NO is $0.12 \mathrm{eV}$. Negative CO adsorption preference, -0.17 $\mathrm{eV}$, indicates stronger adsorption on $\mathrm{Pd}(111)$ than on $\mathrm{Rh}(111)$. Combining these data with TOP descriptors from Table 1 defining surface segregation energy of Pd and Rh atoms on $\{111\}$ nanofacets, $\varepsilon_{T E R R A C E}^{P d}=-\varepsilon_{T E R R A C E}^{R h}$, being respectively ca. -0.54 and $-0.36 \mathrm{eV}$ for $1: 3$ and $1: 1$ 201-atomic Pd-Rh NPs, one can rationalise surface atomic ordering of various $\mathrm{Pd}-\mathrm{Rh}$ NPs in the presence of the chosen adsorbates and predict variation of the ordering with the coverage of the adsorbates. Of course, this simple approach cannot account for surface reconstruction effects expected in case of strong adsorbate-metal 
interactions.

For instance, surface segregation of Rh atoms on the $\{111\}$ terraces of $\mathrm{Pd}_{366} \mathrm{Rh}_{1097} \mathrm{NP}$ to substitute all $234 \mathrm{Pd}$ atoms located there at $0 \mathrm{~K}$ (Table 2) will destabilise the NP by the amount equivalent to the energy gained by adsorption on $\mathrm{Rh}$ terrace sites of 2180 atoms, each stabilising the system by $0.58 \mathrm{eV}$. Thus, ca. 0.5 monolayer (ML) 0 coverage of in total $440\{111\}$ terrace sites exposed on the $\mathrm{Pd}_{366} \mathrm{Rh}_{1097} \mathrm{NP}$ is estimated to be energetically sufficient to make all these metal atomic positions occupied by $\mathrm{Rh}$ atoms. For $\mathrm{Pd}_{731} \mathrm{Rh}_{732}$ and $\mathrm{Pd}_{1097} \mathrm{Rh}_{366} \mathrm{NPs}$, which feature complete Pd shells (Fig. 4), the estimated 0 coverage required to stabilise the orderings with $\mathrm{Rh}$ atoms segregated on all available $440\{111\}$ terrace sites is $\sim 0.6$ ML (or 273 0 atoms). This small coverage increase is due to lower terrace segregation propensity of Pd atoms in case of higher Pd contents.

The outlined above evidences of triggered by adsorbed oxygen Rh surface segregation in Pd-Rh NPs featuring Pd shell arrangement without adsorbates strongly suggests that the experimentally found arrangements of the as-synthesised large Pd-Rh NPs with Rh-rich shells [7] correspond to surface oxidised situations. According to the present calculations, NO shows preference to be adsorbed on Rh rather than Pd sites of Pd-Rh surfaces (although expressed 5 times weaker than that for 0), whereas CO adsorption is preferred on Pd sites vs. Rh ones. These calculated data are in qualitative agreement with the Rh-rich shell structure observed in the presence of NO (or $\mathrm{O}_{2}$ ) adsorbates and substantially increased Pd concentration in the shells in the presence of adsorbed CO [7]. However, the latter experiments have been performed under too complicated conditions, including catalytic ones, to be described in more details by the simplified computational approaches applied in the present study.

\section{Conclusions}

This theoretical modelling study addressed atomic ordering in bimetallic Pd-Rh nanoparticles of different compositions formed of up to more than 3600 atoms. Pd-Rh nanoalloy materials are widely used for applications in catalysis. One of their peculiarities is that the surface composition is very easily adjustable to interactions with adsorbates present in reactive environment. Therefore, the work was focused on detail analysis of the surface composition of bare Pd-Rh nanoparticles and its dependence on the particle size and Pd : Rh stoichiometry.

First, DFT calculations were employed in combination with a novel topological (TOP) approach to quantify atomic ordering and segregation effects in Pd-Rh nanoparticles of $\leq 201$ atoms and $\mathrm{Pd}: \mathrm{Rh}$ compositions $1: 3,1: 1$ and $3: 1$. In all these nanoparticles Pd atoms were notably stabilised in surface positions forming a Pd shell, whereas $\mathrm{Rh}$ atoms preferred to occupy inner positions forming a Rh core. Due to high surface to volume ratio in such particles it was enough Pd atoms to form a complete Pd-shell/Rh-core lowest-energy structure only in the particle $\mathrm{Pd}_{151} \mathrm{Rh}_{50}$ with the highest Pd content. TOP analysis of the DFT data provided quantitative estimates of the energy gained (lost) by appearance of an inner Pd (Rh) atoms in par- ticular surface positions of the studied nanoparticles. This analysis also revealed that the nearest-neighbour $\mathrm{Pd}-\mathrm{Rh}$ contacts (bonds) do not stabilise mixing of these two metal components, in line with experimental observations for bulk materials. Nevertheless, as follows from both DFT and TOP data, at the nanoscale the strong propensity to form the $\mathrm{Pd}$-shell/Rh-core ordering triggers mixing of Pd and Rh atoms with respect to the Janus-type ordering with the most separated compact $\mathrm{Pd}$ and $\mathrm{Rh}$ regions.

Next, the knowledge on the atomic arrangements and the energetics obtained from the DFT and TOP modelling of smaller nanoparticles was used to describe (with DFT accuracy) energetically preferred atomic orderings in the so far inaccessible by DFT $4-5 \mathrm{~nm}$ large Pd-Rh particles approaching the size common for catalytic metal particles. The lowest-energy atomic orderings were determined for $1: 3,1: 1$ and $3: 1 \mathrm{Pd}-\mathrm{Rh}$ fcc crystallites consisting of 1463 and 3630 atoms. The latter, similarly to the aforementioned smaller particles, were found to be energetically the most stable as core-shell structures with the shells consisting of Pd atoms and the cores built of $\mathrm{Rh}$ atoms. Variation of $\mathrm{Pd}: \mathrm{Rh}$ compositions affected the preferred atomic ordering mainly due to the shortage of Pd atoms to form complete Pd shells for the $1: 3$ stoichiometry, resulting in the appearance on the surface terraces of $\mathrm{Pd}_{366} \mathrm{Rh}_{1097}$ and $\mathrm{Pd}_{908} \mathrm{Rh}_{2722}$ particles of $\mathrm{Rh}$ patches affecting surface reactivity. The 1463and 3630-atomic $1: 1$ and $3: 1 \mathrm{Pd}-\mathrm{Rh}$ particles are predicted to expose perfect $\mathrm{Pd}$ shells.

Finally, it was outlined, how the information on atomic ordering in bare bimetallic nanoparticles, in particular, the TOP data, can be used for predicting effects of reacting media on the surface composition and arrangement of the nanoparticles under experimental conditions. For such predictions one usually needs additional data from (often quite intense) calculations of adsorption systems. Employing the TOP analysis makes it possible to estimate surface re-segregation and the resulting surface composition of a nanoalloy in the presence of given amount of adsorbed reactants or intermediates without additional expensive DFT calculations, only using readily available adsorption energies on extended monometallic surfaces. This approach was illustrated for considering surface re-segregation of $\mathrm{Rh}$ in Pd-shell/Rh-core nanoparticles in the presence of adsorbed oxygen, CO and NO reactants. Such simple predictions are expected to be widely applicable to various catalytically important bimetallic particles and to help bridging the gap between idealised surface models and surfaces of technical catalysts.

\section{Acknowledgments}

Work of LV was financed by the Generalitat de Catalunya via a pre-doctoral grant 2018FI-B-00384. HAA is grateful to the Operational program "Science and Education for Smart Growth", project BG05M20P001-2.009-0028 for funding his research stay in the University of Barcelona and for financial support by the Bulgarian Ministry of Education and Science under the National Research Programme "Low-carbon Energy for the Transport and Domestic Use (EPLUS)" approved by 


\section{Graphical Abstract}

Chin. J. Catal., 2019, 40: 1749-1757 doi: S1872-2067(19)63362-0

Using density functional calculations to elucidate atomic ordering of Pd-Rh nanoparticles at sizes relevant for catalytic applications

Lorena Vega, Hristiyan A. Aleksandrov *, Konstantin M. Neyman *

University of Barcelona, Spain; University of Sofia, Bulgaria; Catalan Institution for Research and Advanced Studies (ICREA), Spain

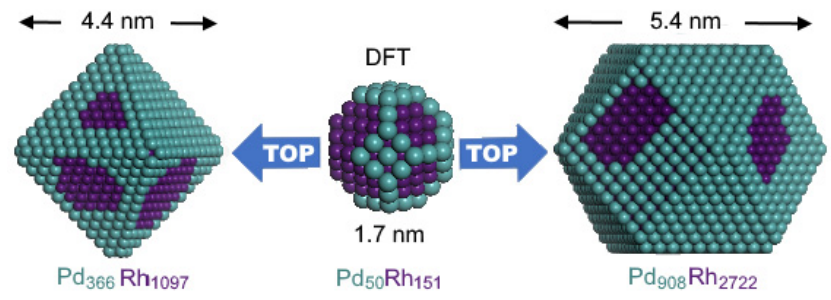

Catalytically active Pd-Rh nanoparticles adapt their surface to chemical environment. Density functional modelling identifies surface composition of Pd-Rh particles containing thousands atoms and paves the way for evaluating surface segregation caused by adsorbed reactants.

DCM \# 577/17.08.2018" (contract D01-214/28.11.2018). KMN acknowledges a support by the Spanish grants PGC2018-093863-B-C22, CTQ2015-64618-R and MDM-20170767 as well as by the grant 2017SGR13 of the Generalitat de Catalunya. LV and KMN thank the Red Española de Supercomputación for providing computer resources and technical support.

\section{Conflicts of interest}

There are no conflicts to declare.

\section{References}

[1] J. Kaspar, P. Fornasiero, N. Hickley, Catal. Today, 2003, 77, 419-449.

[2] H. Goto, K. Komata, S. Minami, SAE Technical Paper 2014-01-1503, 2014, 1-5.

[3] A. A. Vedyagin, M. S. Gavrilov, A. M. Volodin, V. O. Stoyanovskii, E. M. Slavinskaya, I. V. Mishakov, Yu. V. Shubin, Top. Catal., 2013, 56, 1008-1014.

[4] Y.-T. Pan, H. Yang, ChemNanoMat, 2017, 3, 639-645.

[5] C.-T. Hsieh, P.-Y. Yu, D.-Y. Tzou, J.-P. Hsu, Y.-R. Chiu, J. Electroanal. Chem., 2016, 761, 28-36.

[6] H. Idriss, M. Scott, J. Llorca, S. C. Chan, W. Chiu, P.-Y. Sheng, A. Yee, M. Blackford, S. Pas, A. J. Hill, F. M. Alamgir, R. Rettew, C. Petersburg, S. D. Senanayake, M. A. Barteau, ChemSusChem, 2008, 1, 905-910.

[7] F. Tao, M. E. Grass, Y. Zhang, D. R. Butcher, J. R. Renzas, Z. Liu, J. Y. Chung, B. S. Mun, M. Salmeron, G. A. Somorjai, Science, 2008, 322, 932-934.

[8] F. Bernardi, M. E. Grass, Y. P. Hong, R. Chang, N. Jabeen, C. Zhang, B. W. Eichhorn, B. Seo, S. Alayoglu, Z. Hussain, S. H. Joo, Z. Liu, Catal. Today, 2016, 260, 95-99.

[9] P. Müller, U. Hejral, U. Rütt, A. Stierle, Phys. Chem. Chem. Phys., 2014, 16, 13866-13874.

[10] N. J. Divins, I. Angurell, C. Escudero, V. Pérez-Dieste, J. Llorca, Sci- ence, 2014, 346, 620-623.

[11] K. M. Myles, Trans. Met. Soc. AIME, 1968, 242, 1523-1526.

[12] L. H. Bennett, R. E. Watson, CALPHAD, 1981, 5, 19-23.

[13] Y. Oumellal, J.-M. Joubert, C. M. Ghimbeu, J.-M. Le Meins, J. Bourgon, C. Zlotea, Nano-Struct. Nano-Obj., 2016, 7, 92-100.

[14] Yu. V. Shubin, P. E. Plyusnin, S. V. Korenev, J. Alloys Compd., 2015, 622, 1055-1060.

[15] F. Mittendorfer, N. Seriani, O. Dubay, G. Kresse, Phys. Rev. B, 2007, 76, 233413/1-233413/4.

[16] A. V. Ruban, H. L. Skriver, J. K. Nørskov, Phys. Rev. B, 1999, 59, 15990-16000.

[17] K. M. Neyman, R. Sahnoun, C. Inntam, S. Hengrasmee, N. Rösch, J. Phys. Chem. B, 2004, 108, 5424-5430.

[18] R. Ferrando, J. Jellinek, R. L. Johnston, Chem. Rev., 2008, 108, 845-910.

[19] L. Cao, C. Li, T. Mueller, J. Chem. Inf. Model., 2018, 58, 2401-2413.

[20] J. M. Sanchez, F. Ducastelle, D. Gratias, Phys. A, 1984, 128, 334-350.

[21] L.-L. Wang, T. L. Tan, D. D. Johnson, Nano Lett., 2014, 14, 7077-7084.

[22] I. V. Yudanov, R. Sahnoun, K. M. Neyman, N. Rösch, J. Chem. Phys., 2002, 117, 9887-9896.

[23] A. Roldán, F. Viñes, F. Illas, J. M. Ricart, K. M. Neyman, Theor. Chem. Acc., 2008, 120, 565-573.

[24] S. M. Kozlov, K. M. Neyman, Top. Catal., 2013, 56, 867-873.

[25] S. M. Kozlov, G. Kovács, R. Ferrando, K. M. Neyman, Chem. Sci., 2015, 6, 3868-3880.

[26] G. Kovács, S. M. Kozlov, K. M. Neyman, J. Phys. Chem. C, 2017, 121, 10803-10808.

[27] G. Kresse, J. Furthmüller, Phys. Rev. B, 1996, 54, 11169-11186.

[28] G. Kresse, J. Hafner, Phys. Rev. B, 1994, 49, 14251-14269.

[29] J. P. Perdew, K. Burke, M. Ernzerhof, Phys. Rev. Lett., 1996, 77, 3865-3868; Phys. Rev. Lett., 1997, 78, 1396-1396.

[30] P. E. Blöchl, Phys. Rev. B, 1994, 50, 17953-17979.

[31] G. Kresse, D. Joubert, Phys. Rev. B, 1999, 59, 1758-1775.

[32] F. Viñes, F. Illas, K. M. Neyman, Angew. Chem. Int. Ed., 2007, 46, 7094-7097.

[33] G. Kovács, S. M. Kozlov, I. Matolínová, M. Vorokhta, V. Matolín, K. 
M. Neyman, Phys. Chem. Chem. Phys., 2015, 17, 28298-28310.

[34] A. Wolfbeisser, G. Kovács, S. M. Kozlov, K. Föttinger, J. Bernardi, B. Klötzer, K. M. Neyman, G. Rupprechter, Catal. Today, 2017, 283, 134-143.

[35] R. Ferrando, A. Fortunelli, G. Rossi, Phys. Rev. B, 2005, 72, 085449/1-085449/9.

[36] I. V. Yudanov, K. M. Neyman, Phys. Chem. Chem. Phys., 2010, 12,
5094-5100.

[37] M. Mamatkulov, I. V. Yudanov, A. V. Bukhtiyarov, I. P. Prosvirin, V. I. Bukhtiyarov, K. M. Neyman, J. Phys. Chem. C, 2019, 123, 8037-8046.

[38] S. Liu, Z.-J. Zhao, C. Yang, S. Zha, K. M. Neyman, F. Studt, J. Gong, ACS Catal., 2019, 9, 5011-5018.

\title{
利用密度泛函计算阐明Pd-Rh纳米颗粒在与催化应用相关尺寸下的原子排序 \\ Lorena Vega ${ }^{\text {a }}$, Hristiyan A. Aleksandrov ${ }^{\text {b,* }}$, Konstantin M. Neyman ${ }^{\text {a,c,\# }}$ \\ 巴巴塞罗那大学材料科学与化学物理系, 理论化学计算研究所, 巴塞罗那, 西班牙 \\ $\mathrm{b}^{\mathrm{w}}$ 索非亚大学化学与药学系, 索非亚, 保加利亚 \\ c加泰罗尼亚自治大学, 巴塞罗那, 西班牙
}

\begin{abstract}
摘要: 从所周知, Pd-Rh纳米颗粒在反应环境中容易发生表面再构. 本文借助密度泛函(DFT)计算和一种新的拓扑方法, 对 组成分别为 $1: 3 、 1: 1$ 和 $3: 1$ 的(多达 201 个原子, 约 $1.7 \mathrm{~nm}$ )的Pd-Rh粒子中的原子排序和表面偏析效应进行了定量研究. 所得 数据用于可靠优化用DFT无法研究的、含有数千个原子且尺寸超过 $5 \mathrm{~nm}$ 的Pd-Rh粒子能量优先原子排序, 这是典型的催化金 属粒子. 概述了在现有的模拟装置中, 如何通过简单的方法评估偏析效应对吸附质诱导的Pd-Rh纳米合金催化剂表面排列 的影响.
\end{abstract}

关键词: 密度函数计算; 原子序; Pd-Rh纳米粒子; 尺寸相关

收稿日期: 2019-03-02. 接受日期: 2019-04-06. 出版日期: 2019-11-05.

*通讯联系人. 电子信箱: haa@chem.uni-sofia.bg

\#通讯联系人. 电子信箱: konstantin.neyman@icrea.cat

本文的电子版全文由Elsevier出版社在ScienceDirect上出版(http://www.sciencedirect.com/science/journal/18722067).

For Author Index:

VEGA Lorena, ALEKSANDROV Hristiyan A., NEYMAN Konstantin M. 